\title{
In Silico Designing and Interaction of Coumarin-Amino Acid(s) Conjugates with Integrin Like Protein of Cryptococcus neoformans: Insights on Antifungal Drug Design
}

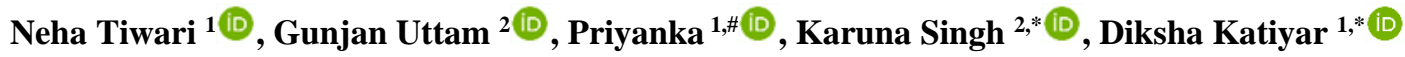 \\ 1 Department of Chemistry, MMV, Banaras Hindu University, Varanasi-221005 \\ 2 Department of Zoology, MMV, Banaras Hindu University, Varanasi-221005 \\ \# Present address: Department of Chemistry, Indian Institute of Technology Ropar, Rupnagar, Punjab 140001, India \\ * Correspondence: karunazoobhu@gmail.com (K.S.); dikshakatiyar@ gmail.com (D.K.);
}

Scopus Author ID 6602656152

Received: 3.07.2020; Revised: 30.07.2020; Accepted: 31.07.2020; Published: 2.08.2020

\begin{abstract}
In the present work, a library of 117 coumarin-amino acid(s) conjugates was designed, and molecular docking study was performed to investigate their possible role as fungal integrin like receptor antagonists. The objective of this study is in-silico designing and docking of coumarin-amino acid conjugates against integrin like protein of Cryptococcus neoformans. In the absence of a crystallographic structure of integrin of the fungal pathogen, a homology model of protein OXH63806.1 of Cryptococcus neoformans was developed using the currently available X-ray structure of human integrin as a template. The quality of the 3D model obtained by homology modeling was evaluated by the PROCHECK program. A docking study using coumarin-amino acid(s) conjugates as ligands on the binding site of the modeled receptor was carried out to understand the protein-ligand binding interactions. Some of the compounds have shown very good binding energies ranging from -10.32 to $10.94 \mathrm{Kcal} / \mathrm{mol}$ towards the target receptor. These results may be helpful in the designing and development of new antifungal agents as integrin like protein antagonists.
\end{abstract}

Keywords: Antifungal; Cryptococcus neoformans; Drug Design; integrin; RGD mimetics; Homology modeling.

(C) 2020 by the authors. This article is an open-access article distributed under the terms and conditions of the Creative Commons Attribution (CC BY) license (https://creativecommons.org/licenses/by/4.0/).

\section{Introduction}

The past few decades have witnessed the dramatic rise in fungal infections. It is estimated that approximately 1.2 billion of the world population suffers from these infections [1]. Alarmingly, these infections cause approximately 1.3 million deaths annually worldwide, exceeding those killed by malaria or tuberculosis [2,3]. The current arsenal of antifungal drugs consisting of four classes of antifungal agents targets a limited number of cellular processes. Based on the mode of action, these are: polyenes (nystatin and amphotericin B) bind to ergosterol; echinocandins (e.g., caspofungin and micafungin) target the protein complex responsible for the synthesis of $\beta-1,3$ glucans by blocking the enzyme glucan; azoles (e.g., fluconazole, voriconazole, ketoconazole, itraconazole, and posaconazole) target the ergosterol biosynthetic enzyme lanosterol demethylase; and nucleoside analogs (e.g.,5-flucytosine) inhibit the nucleic acid synthesis [4]. The current armamentarium of antifungals has several limitations, such as toxicity to hosts, development of drug resistance, and fungistatic activity 
[5-7]. Thus, the development of new antifungal drugs against the novel target is highly desirable in the health care field.

The phylogenetic relatedness between fungi and humans offers relatively few differential targets that can be exploited for developing drugs against fungal infections. Like mammalian cells, fungi are also eukaryotes, and thus agents that inhibit protein synthesis, RNA, and DNA biosynthesis have greater potential for toxicity [8, 9]. However, the fungal cell wall represents an excellent target for antifungal compounds because of its absence in human cells $[10,11]$. The cell wall provides protection to the fungus and facilitates its interaction with the host. [12]. The modification of host-fungi interactions by impeding putative virulence factors is an emerging area of research in antifungal chemotherapy [13]. In recent years, several molecules with surface integrin-like receptors, called adhesins (or fungal integrins), have been described in pathogenic fungi, which play an important role in host-pathogen interaction, including adherence and dissemination of infection [14, 15]. Like mammalian integrins, RGD (arginine-glycine-aspartic acid) sequence containing molecules and peptidomimetic compounds has shown to inhibit fungal binding to endothelial cells [16-21]. An integrin-like receptor protein known as $\alpha$ Int1p has been identified in C. albicans [22] that shares structural similarity and sequence homology to the $\alpha$-subunits of human leukocyte integrins. This protein contains RGD extracellular binding site and helps in the attachment of fungi to epithelial cells in the host to initiate the infection process. Some of the recent studies have also shown that inhibition of integrin like proteins in fungi can lead to the prevention of infection [23-26]. Therefore, the development of integrin antagonists may find a beneficial application in the treatment of life-threatening fungal infections.

In this direction and in continuation of our work on the synthesis of bioactive molecules, $[27,28]$ we envisaged to design a library of compounds containing short peptides and perform their molecular docking studies into the active site of integrin like protein of fungi and investigate their antifungal properties as integrin antagonists. Coumarins are a well-known group of compounds that display a wide range of biological activities such as antimicrobial, anti-inflammatory, and anticancer activities [29]. Recently, many reports have been published in literature depicting the antifungal effect of the coumarin pharmacophore [30-33]. Therefore, for docking experiments, we designed a library of 117 coumarin-amino acid(s) conjugates (monopeptides, dipeptides, and tripeptides). In the absence of a crystallographic structure of fungal integrin, we developed a homology model of hypothetical protein J000_06513 and entry name OXH63806.1 from Cryptococcus neoformans var. grubii using the currently available $\mathrm{X}$-ray structure of human integrin as a template. It is hoped that the homology model and ligand-receptor binding interactions reported herein may be useful for the rational design of novel fungal integrin antagonists.

\section{Materials and Methods}

\subsection{Sequence retrieving, analysis, and homology modeling.}

The 3D structure of human pathogenic fungal integrin is not available in Protein Data Bank (PDB: www.rcsb.org). Therefore the homology modeling was used to determine the 3D structure of fungal integrin [34,35]. NCBI (National Center of Biotechnology Information, www.ncbi.nlm.nih.gov) protein database search using keyword fungi + integrin showed 603 sequences. The amino acid sequences of integrin of some pathogenic fungi such as Cryptococcus sp., Fusarium sp., Aspergillus sp., and Candida sp.with accession number 
OXH63806.1 (hypothetical protein), PYH75478.1, KPA40816.1 and XP_019330964.1 (Int1P), respectively, were retrieved from NCBI in fasta format. The homology modeling of $3 \mathrm{D}$ structure of retrieved sequences of integrins using $\mathrm{Phyre}^{2}$ server (www.sbg.bio.ic.ac.uk/ phyre ${ }^{2} /$ ) showed coverage $\%$ and confidence $\%$ as follows: $46 \%$ and 100\% for OXH63806.1; 28\% and 100\% for PYH75478.1; 20\% and 99.8\% for KPA40816.1 and 15\% and 100\% for XP_019330964.1. OXH63806.1 was identified as the best target protein based on the coverage or sequence identity (46\%) between query and template protein sequences. The template c4um9D (chain D, PDB header-immune system, PDB moleculeintegrin $\beta-6$ ) was used by Phyre $^{2}$ to model the 3D structure of the hypothetical protein with accession number OXH63806.1. This query sequence contains metal ion-dependent adhesion site (MIDAS), integrin-collagen binding site, and integrin inhibitor binding pocket as conserved domains. The active site of the resultant model was predicted by using SCFBio tools, and the validity of this model was checked by PROCHECK [36].

\subsection{Ligand set-up.}

Ligand 3D structure was drawn using ACD/ChemSketch [37]. First, the 2D structures of the ligands were drawn. They were then converted into the 3D structure (mol file). The 3D structure in mol form was converted into .pdb format using OpenBabelGUI software.

\subsection{Molecular docking.}

Molecular docking was performed using AutoDockTools (ADT) version 1.6.6rc3 and AutoDock version 4.0 docking program [38]. The structure of the model OXH63806.1 was used as a receptor for docking studies. Initially, before docking, polar hydrogen was added, and Kollman charges were added to each atom. Then the structure was saved in .pdbqt format. 3D structures of designed ligands were drawn using ACD/ChemSketch. After this, all the structures were saved in .pdb format using OpenBabelGUI. So as to carry out the docking of the ligands, they were saved in .pdbqt format in ADT. A grid box with dimension $40 \times 40 \times 40$ $\AA^{3}$ with $0.703 \AA$ spacing and centered on $20.321,21.012$ and 77.780 was created around the ligand binding of the integrin. For the docking calculations, the default parameters of AutoDock were used, and for each compound, 10 docked conformations were generated. The energy calculations were done using genetic algorithms. Docked ligand conformations were analyzed in terms of binding energy and hydrogen bond interactions between the ligand and the receptor integrin. For the display of the receptor with the ligand-binding site, Chimera $1.11[39]$ was used.

\section{Results and Discussion}

\subsection{Homology modeling.}

The quality of the 3D model of OXH63806.1 (Fig. 1) obtained by homology modeling was evaluated by the PROCHECK program. The Ramachandran plot obtained showed that the majority of the amino acid residues were in the most favored region with a percentage of $90.2 \%$, $8.2 \%$ residues were in additional allowed regions, $1.1 \%$ residues were in the generously allowed region, and only $0.5 \%$ residues were in the disallowed region. The Ramachandran plot obtained by PROCHECK is shown in Fig. 2. 


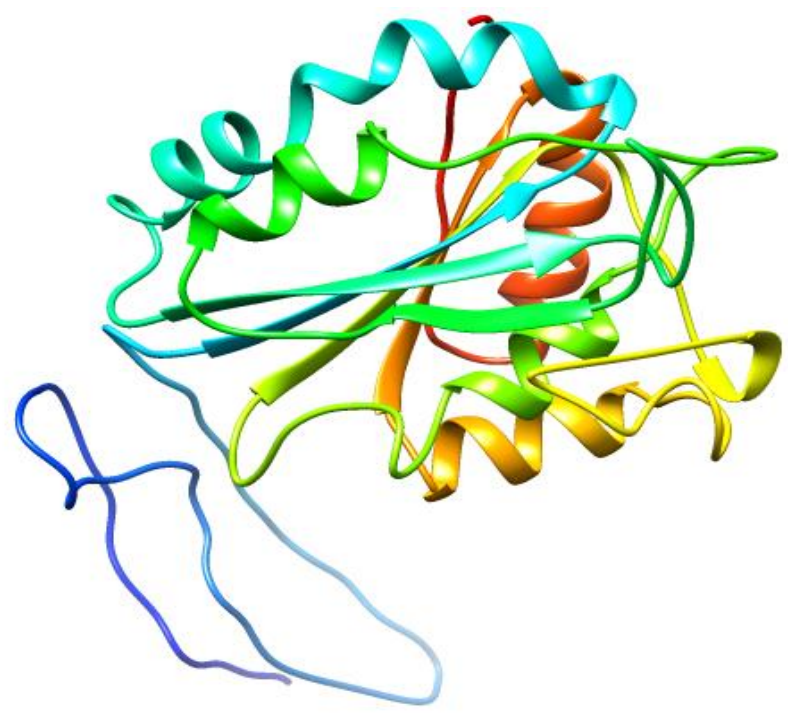

Figure 1. Modeled structure of OXH63806.1 of Cryptococcus neoformans.

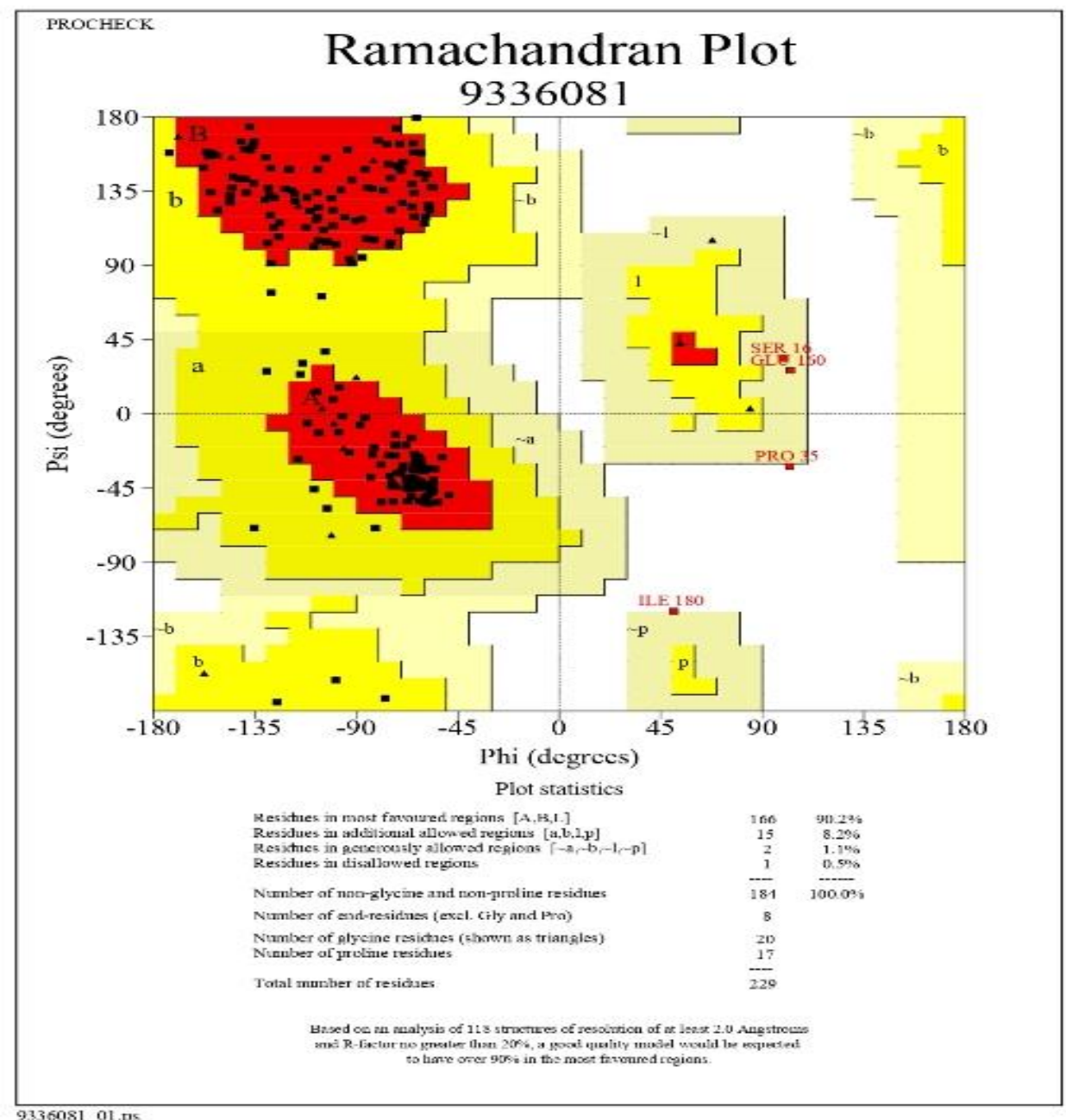

Figure 2. Ramachandran plot.

Additionally, the main chain parameter plot was also generated. The properties represented in this plot are (i) Ramachandran plot quality assessment, (ii) peptide bond planarity, (iii) bad non-bonded interaction, (iv) alpha carbon tetrahedral distortion, (v) hydrogen bond energies and (vi) overall G-factors. The results of this plot showed that out of the six properties, five of them represent the solid square inside the dark band of each graph, 
and only one property showed it outside. From this, it is clear that our model shows good stereochemistry and can be used for ligand-protein docking studies. The Main Chain Parameter plot obtained by PROCHECK is depicted in Fig. 3.

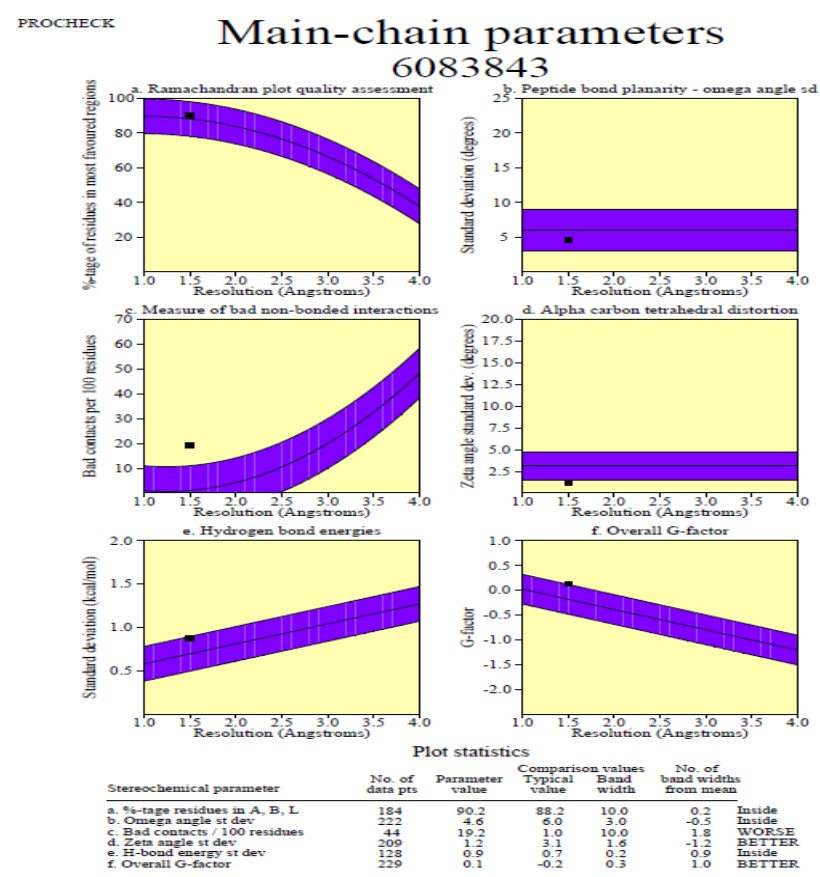

Figure 3. Main chain parameter plot.

\subsection{Active site prediction.}

The active site was predicted using SCFBio tools. From this tool, nine different cavities were found to be active. Since most of the monopeptides were bound to cavity 3 , hence cavity 3 with amino acid sequence Pro, Gly, Arg, Asp, Met, Leu, Ile, Cys, Ala, Val, His, Thr, Phe, Ser, Tyr (PGRDMLICAVHTFSY) was considered as the most active one.

\subsection{Ligand design and molecular docking studies.}

With the aim to develop short peptides with potent antifungal activity, we designed a library of 117 coumarin-amino acid(s) conjugates. For designing of the compounds, we chose nine amino acids: Gly, Ala, and Lue with nonpolar, aliphatic ' $\mathrm{R}$ ' group; Asp with negatively charged ' $R$ ' group; Arg and His with positively charged ' $R$ ' group; Phe and Trp with aromatic ' $R$ ' group and Pro with aliphatic cyclic ' $R$ ' group for large sequence diversity and linked them to the 3-amino coumarin nucleus by an amide linkage to obtain nine coumarin-monopeptide conjugates L01-L09 (Fig. 4). Firstly, all the monopeptides were blindly docked on the modeled receptor using AutoDock. The docking results showed that most of the compounds were bound to cavity 3. The active site consisted of amino acid residues Pro243, Gly81, Arg77, Met168, Leu58, Ile79, Cys83, Val170, Val214, His78, Tyr71, Phe212, Thr245, and Ser74. Out of the nine monopeptides docked, three top-ranked compounds L05, L07, and L08 bearing Arg, Trp, and His as amino acid residues, respectively, were selected for further study (Table 1). Next, we designed dipeptides by sequentially attaching Gly, Ala, Leu, Asp, Arg, Phe, Trp, His, and Pro residues to each L05, L07, and L08 to obtain twenty-seven dipeptides with coumarin fragment. All the designed dipeptides were again docked on the same particular grid, and their binding energy was calculated. The structures of the top-ranked three ligands in each group showing the lowest binding energy (Table 1) are shown in Fig. 5. The binding energy of other 
dipeptide ligands is given in supplementary information. Furthermore, to check the effect of the addition of third amino acid on activity profile, we attached the amino acid Gly, Ala, Leu, Asp, Arg, Phe, Trp, His, and Pro to each dipeptide L10-L18 sequentially and designed the short library of total eighty-one tripeptides. The designed ligands were also docked on the modeled receptor, and the binding energy was calculated. The structures and binding energy of the top eight compounds L19-L26 are shown in Fig.6 and Table 1, respectively. The binding energy of other tripeptides is provided in the supplementary information. To see the inhibitory property of RGD sequence, we also designed anL27 having RGD sequence linked with coumarin moiety. The cyclic peptides are also reported to have a beneficial effect on the integrin inhibitory activity profile [40, 41]. Thus, L21 and L23 bearing carboxylate group at one end were linked to the amino group at the position seven of the coumarin ring by an amide bond to obtain cyclic peptides L28 and L29 and their binding energy was calculated (Table 1, Fig. 7).

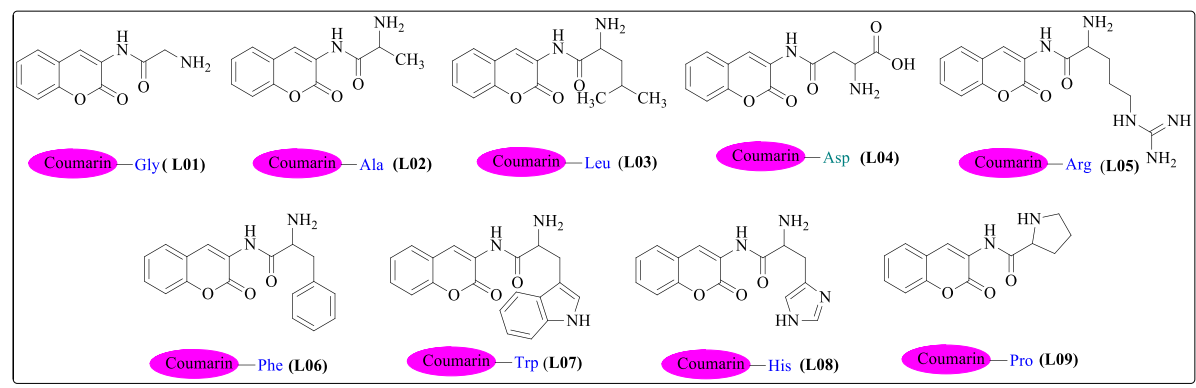

Figure 4. Structures of coumarin-monopeptide conjugates.

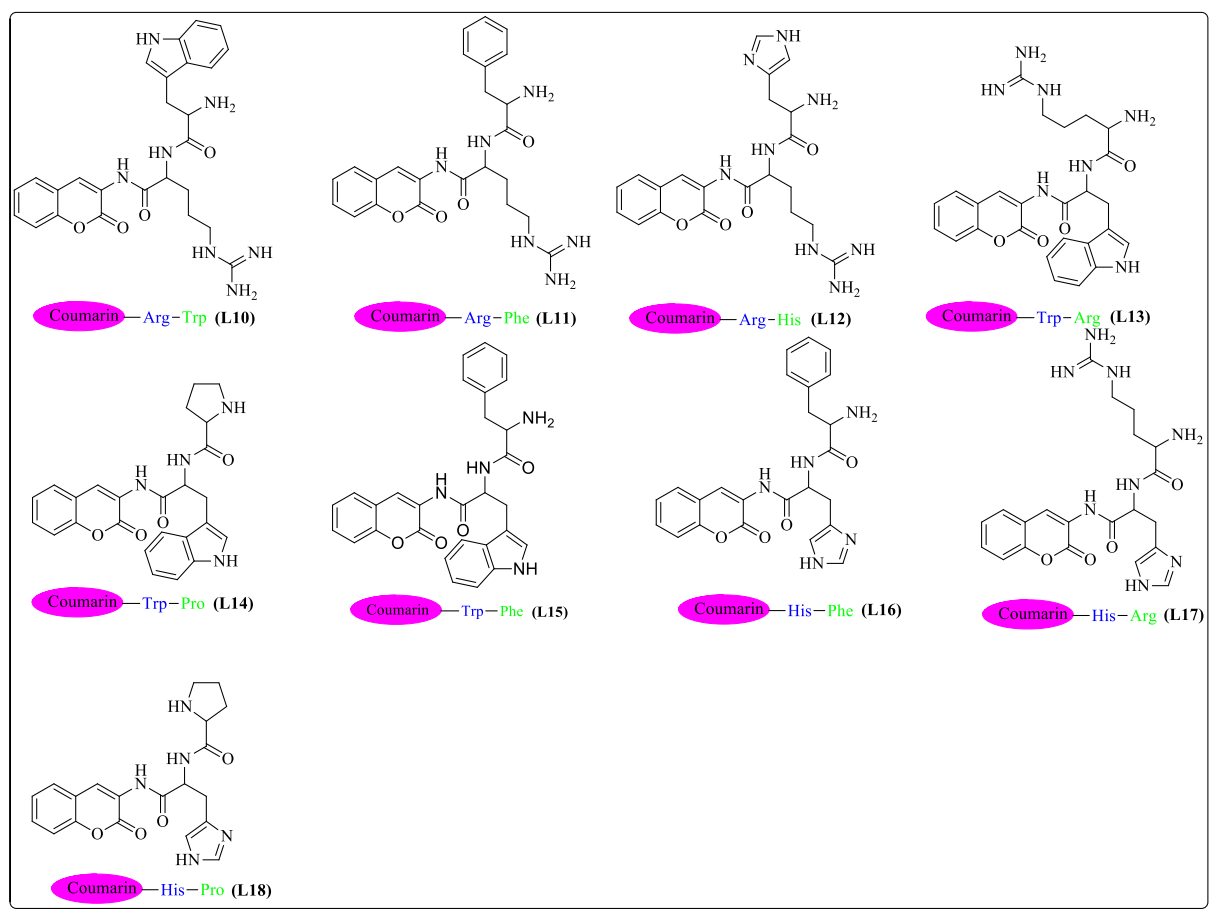

Figure 5. Structures of coumarin-dipeptide conjugates.

Detailed analysis of docking results revealed that binding energy was affected both by the nature and number of the amino acid residues. The binding affinity increased (lower value) from monopeptides $(-7.22$ to $-5.43 \mathrm{Kcal} / \mathrm{mol})$ to dipeptides $(-10.58$ to $-7.81 \mathrm{Kcal} / \mathrm{mol})$, and the lowest binding energy was obtained with tripeptides $(-10.94$ to $-10.32 \mathrm{Kcal} / \mathrm{mol})$. All the monopeptides showed binding energy higher than amphotericin B $(-10.22 \mathrm{Kcal} / \mathrm{mol})$, among 
dipeptides only L10 and among tripeptides only nine ligands L19-L27 showed binding energy lower than amphotericin B.

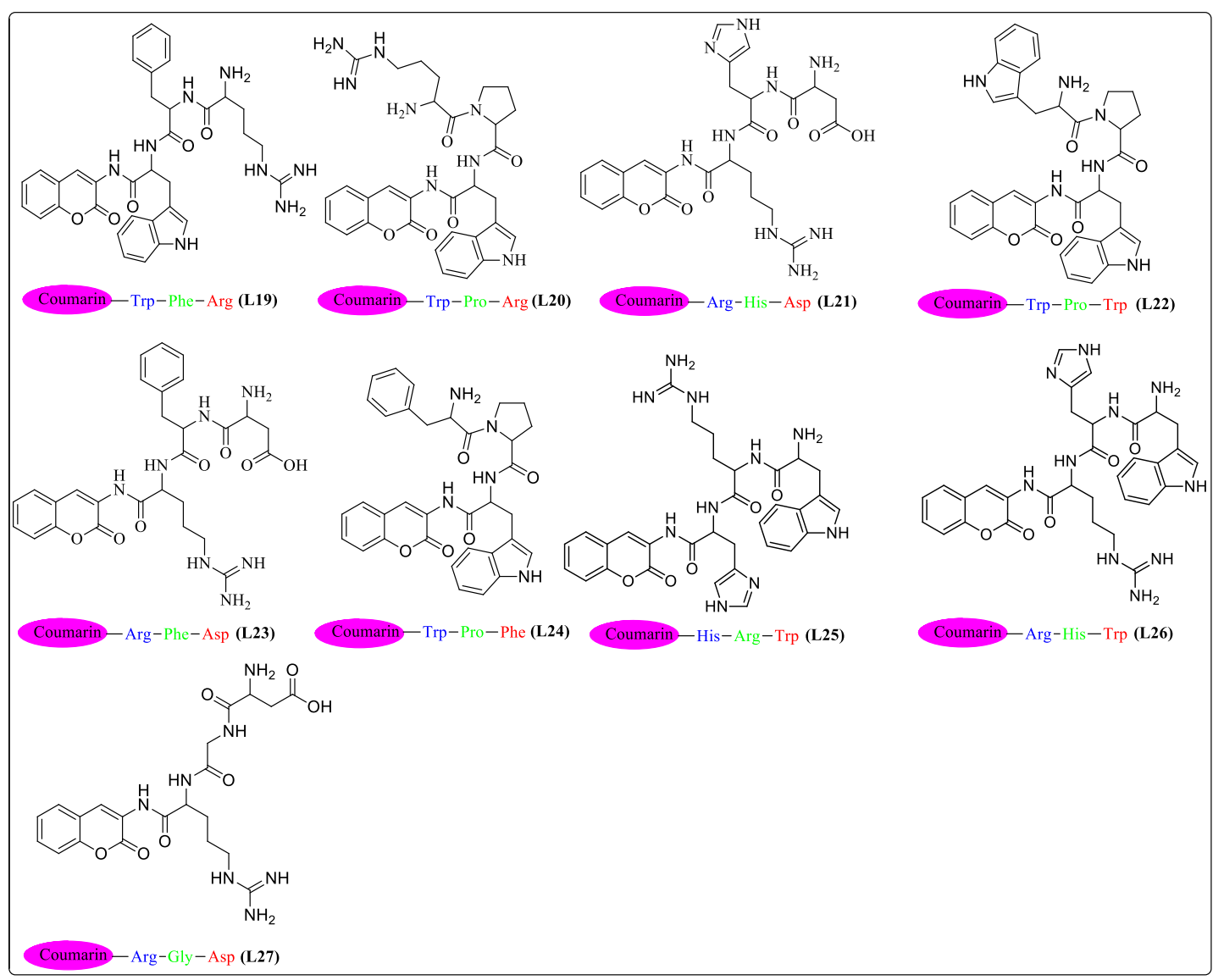

Figure 6. Structures of coumarin-tripeptide conjugates.

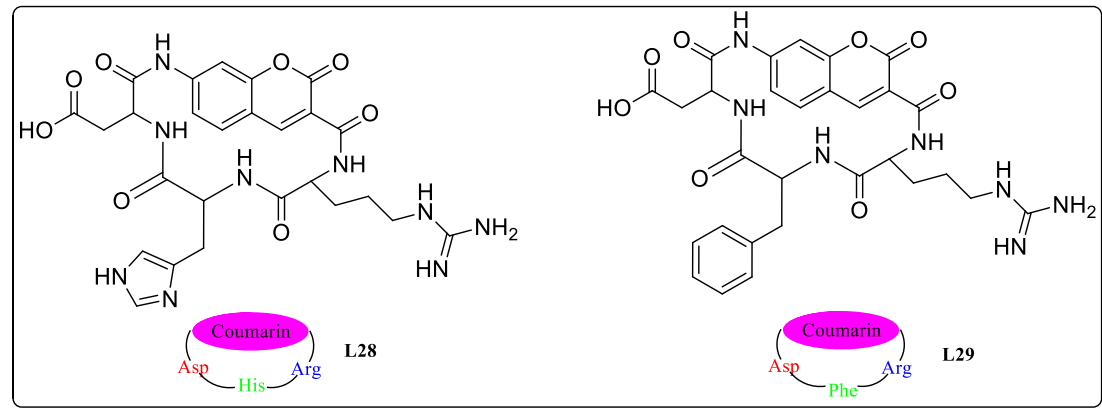

Figure 7. Structures of cyclic peptide incorporating coumarin.

Table 1. Docking results of coumarin-amino acid(s) conjugates.

\begin{tabular}{l|l|l|l|l|l|l|l|l} 
Ligand & B.E & $\begin{array}{l}\text { H- } \\
\text { bonds }\end{array}$ & Ligand & $\mathbf{B . E}$ & $\begin{array}{l}\text { H- } \\
\text { bonds }\end{array}$ & Ligand & $\begin{array}{l}\text { B.E } \\
\text { Kcal/mol }\end{array}$ & H-bonds \\
\hline L05 & -7.22 & 1 & L10 & -10.58 & 4 & L19 & -10.94 & 3 \\
\hline L07 & -6.94 & 3 & L13 & -9.99 & 1 & L20 & -10.89 & 3 \\
\hline L08 & -6.38 & 1 & L14 & -9.55 & 3 & L21 & -10.87 & 0 \\
\hline L02 & -6.28 & 2 & L15 & -9.39 & 2 & L22 & -10.85 & 1 \\
\hline L06 & -6.26 & 0 & L16 & -8.12 & 2 & L23 & -10.76 & 0 \\
\hline L09 & -6.04 & 2 & L17 & -8.09 & 1 & L24 & -10.64 & 0 \\
\hline L01 & -6.02 & 1 & L11 & -7.88 & 2 & L25 & -10.36 & 0 \\
\hline L03 & -5.97 & 1 & L18 & -7.87 & 4 & L26 & -10.32 & 5 \\
\hline $\mathbf{L 0 4}$ & -5.43 & 1 & L12 & -7.81 & 0 & L27 & -10.32 & 0 \\
\hline & & & & & & L28 & -7.75 & 3 \\
\hline & & & & & & L29 & -7.82 & 3 \\
\hline & & & & & & Amphotericin B & -10.22 & 2
\end{tabular}


The important interactions existed in L10 and L19-L23 are summarized in Table 2. In L10, which showed binding energy value of $-10.58 \mathrm{Kcal} / \mathrm{mol}$, the Arg and the Trp side chains of the ligand pointed to opposite directions. The Arg group on the one side formed a hydrogen bond with the hydroxyl group of Thr245, and on the opposite side aromatic ring of Trp residue formed $\pi$ stacking interactions with Tyr71. The amide carbonyl oxygen and the amino group of Trp residue also formed hydrogen bonds with Thr246 and Asp248 residues of the receptor, respectively. The carbonyl oxygen of the coumarin ring formed a hydrogen bond with the hydroxyl group of Try71, and the coumarin ring was stabilized by strong hydrophobic interactions with nonpolar residues Val170, Leu 58, Ile 82, Phe 60 and Val172 residues (Fig. $8)$.

The tripeptide L19 with the lowest free energy of binding $(-10.94 \mathrm{Kcal} / \mathrm{mol})$ among all the designed ligands showed three hydrogen bond interactions with the receptor. As shown in Fig. 8, the amino groups of terminal Arg residue displayed hydrogen bond interactions with a side chain of Tyr71 while the amide amino hydrogen of Phe residue formed a hydrogen bond with a side chain of Pro243. The coumarin moiety was stabilized by hydrophobic interactions with Phe60, Val75, Ser74, and His78 residues while the amino acid residues Trp-Phe-Arg formed strong hydrophobic interactions with Ile82, His78, Cys83, Val170, Val172, Phe212, Phe60, and Leu62. The high binding affinity and number of observed hydrogen bonding interactions between the receptor and the ligand at the active site enabled L19 to be a strong binding inhibitor. In contrast, L20-L26 were found to be less stabilized in the active site as compared to L19 and exhibited low binding affinity with binding energy values of -10.89 , $10.87,-10.85,-10.76,-10.64,-10.36$ and $-10.32 \mathrm{kcal} / \mathrm{mol}$, respectively. L20 showed three hydrogen bond interactions with the receptor. One hydrogen bond was observed between the carbonyl oxygen of coumarin moiety and $\mathrm{NH}$ group of His78 with bond distance $2.774 \AA$; second hydrogen bond interaction was between the amide carbonyl oxygen of Pro residue of ligand and hydroxyl of Tyr71 with a bond distance of $3.31 \AA$ and the third hydrogen bond interaction was between NH group of Trp and hydroxyl group of Thr246 with a bond distance of 1.970 ̊.L22 showed only one hydrogen bond. L21, L23-L25 did not show any hydrogen bond interactions with the receptor, while L26 instead of the formation of five hydrogen bonds with the receptor showed binding energy higher than that of L19. In this study, the effect of cyclic peptides on the inhibitory potential of ligands was also observed. L21 and L23 having a carboxylate group at one end were linked to the amino group at the position seven of the coumarin ring by an amide bond. Interestingly, cyclic ligands L28 and L29 showed binding energy approximately $3.0 \mathrm{Kcal} / \mathrm{mol}$ higher than that of their non-cyclic analogs L21 and L23. It is noteworthy that L27 with the RGD sequence did not fit very well in the active site and had binding energy higher than the L19-L24.

By looking at the tripeptide series, it is interesting to note that the higher affinity of L19 could be ascribed to its symmetric orientation in the active site of the receptor, the coumarin moiety and the terminal Arg group were orientated on the same side and are stabilized by hydrophobic interactions and hydrogen bond, respectively. At the same time, the side chains of Phe and Trp residues were orientated in the opposite direction and were stabilized by hydrophobic interactions. Additionally, analysis of the docking sites revealed that the binding of L19 was augmented by $\pi$-stacking interactions between the aromatic ring of coumarin moiety and Phe60 of the receptor and also between His78 of the receptor and side chain of Trp residue of the ligand (Fig. 9b). 
A literature survey revealed that the number of antifungal peptides (AFPs) and antimicrobial peptides (AMPs) exhibiting antifungal activity contain Trp, Arg, His, Phe, Lys and Leu amino acids in common and in abundance. The presence of these amino acids and sometimes their repeat in specific sequences play a pivotal role in the antifungal profile of the peptides [41-43]. Notably, out of all the compounds designed in the present study, the most active compounds (L19-L27) were also found to contain tripeptide sequences comprising mainly of amino acids Trp, Arg, His, and Phe. Taken together, some coumarin-tripeptide conjugates, especially L19 have demonstrated the high binding affinity with the receptor and may prove useful for the designing and development of new compounds capable of inhibiting integrin like proteins of fungal pathogens, although experimental validation of the interaction mechanism between ligands and receptor is further needed.
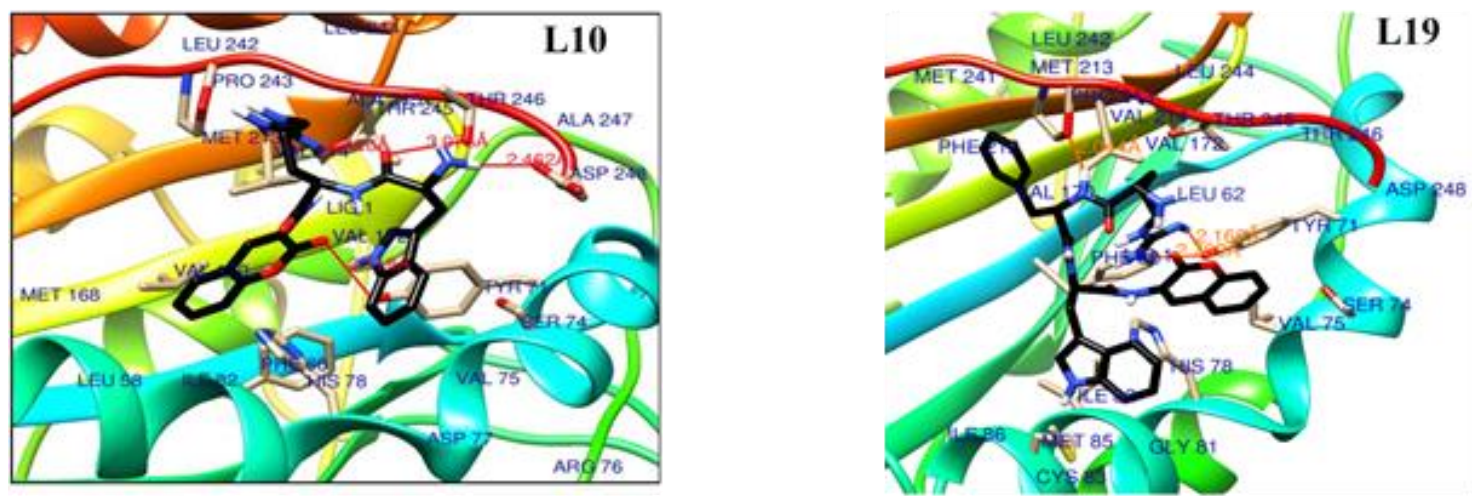

Figure 8. Binding mode of L10 and L19 in the active site of modeled receptor.
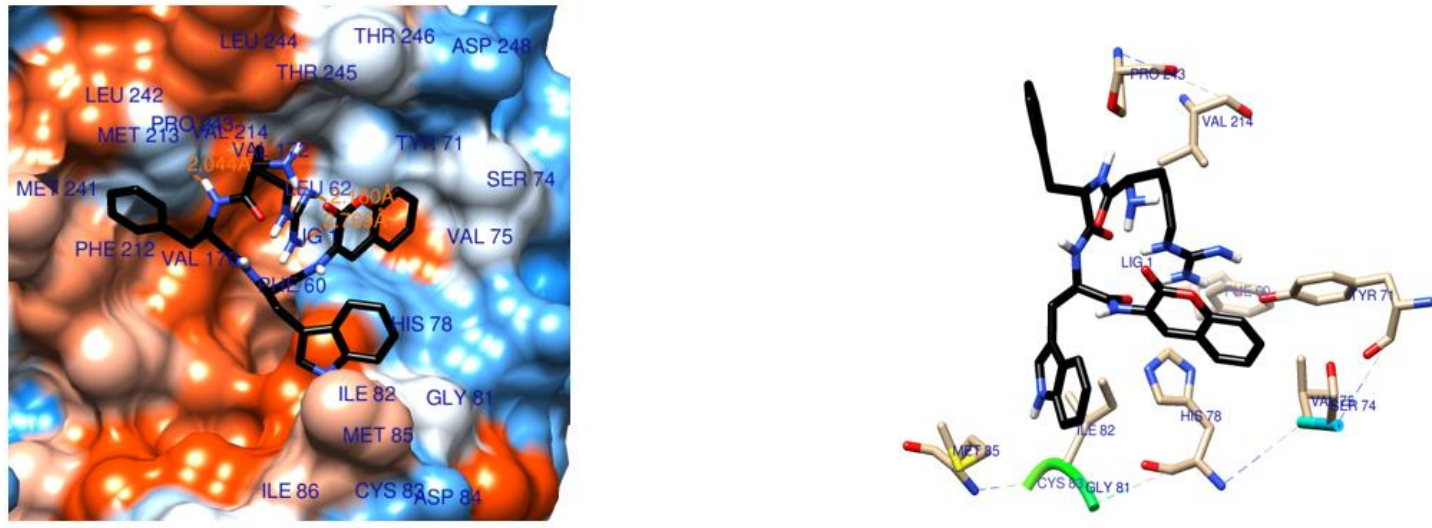

Figure 9. (a) Binding mode of L19 in hydrophobic surface (left), (b) Interaction of L19 with amino acids of the active site (right).

Table 2 Major interactions of ligands with the receptor*.

\begin{tabular}{c|c} 
Ligand & $\begin{array}{c}\text { Surrounding Residues } \\
\text { L10 }\end{array}$ \\
\hline L19 & $\begin{array}{r}\text { Leu58, Phe60, Tyr71(H-bond), Ser74, Val75, His78, Met168, Val170, Val172, Met213, Val214, Pro243, } \\
\text { Thr245(H-bond), Thr246 (H-bond), Asp248(H-bond) }\end{array}$ \\
\hline L20 & $\begin{array}{r}\text { Leu58, Phe60, Tyr71(H-bond), Ser74, His78(H-bond), Ile78, Met168, Val170, Val172, Phe212, Val214, } \\
\text { Phe212,Val214,Pro243(H-bond) } \\
\text { Pro243, Thr245, Thr246(H-bond) }\end{array}$ \\
\hline L21 & Ile56,Leu58,Phe60,Ile82,Met85,Ile86,Gly94,Met168,Val170,Val172,Phe212 \\
\hline L22 & Leu58,Phe60,Try71,His78,Ile82,Met85,Met168,Phe212,Val170,Val172,Thr245,Thr246 \\
\hline L23 & Lys54,Ile56,Leu58,Phe60,Ile82,Met85,Ile86,Leu98,Ala166,Met168,Val170,Val172,Phe212
\end{tabular}

*The images for L20-L23 are provided in the supplementary information. 


\section{Conclusions}

Here in this study, a library of coumarin-amino acid conjugates was designed, and the molecular docking study was carried out to explore the lead compound. In the absence of Xray crystal structure of integrin of fungi, homology modeling was performed to model a suitable three-dimensional structure of OXH63806.1 of C. neoformans, which contains metal iondependent adhesion site (MIDAS), integrin-collagen binding site and integrin inhibitor binding pocket as conserved domains. Since the pdb structure of integrin was not available, and we have done homology modeling, so there is no need for further optimization of the receptor, and hence the E-values were not found. This modeled structure was used as a receptor to perform docking. After analyzing the results, it was found that coumarin-tripeptide conjugates showed the best binding energies as compared to monopeptides and dipeptides. However, these theoretical findings are to be confirmed by experimental tests. The detailed analysis of the docked ligands with the protein has pointed out some major interaction sites and key amino acid residues in the binding pocket, which can be taken into account for the design of new compounds. The amino acid residues Tyr71, Thr245, Thr246, Val75, Asp77, His78, and Phe60 play an important role in stabilizing the ligand into the active site of the receptor. The synthesis and studies related to the biological activity of designed compounds are underway in our laboratory, and the results will be reported in due course.

\section{Funding}

This study was funded by the Science and Engineering Research Board (SERB), India (grant number: EMR/2016/001396).

\section{Acknowledgments}

This research has no acknowledgment.

\section{Conflicts of Interest}

The authors declare no conflict of interest.

\section{References}

1. Denning, D.W.; Bromley, M.J. How to bolster the antifungal pipeline. Science 2015, 347, 1414-1416, https://doi.org/10.1126/science.aaa6097.

2. Brown, G.D.; Denning, D.W.; Gow, N.A.R.; Levitz, S.M.; Netea, M.G.; White, T.C. Hidden killers: human fungal infections. Science Translational Medicine 2012, 4, https://doi.org/10.1126/scitranslmed.3004404.

3. www.gaffi.org

4. Gintjee, T.J.; Donnelley, M.A.; Thompson, G.R. Aspiring antifungals: review of current antifungal pipeline developments. Journal of Fungi 2020, 28, 1-11, https://doi.org/10.3390/jof6010028.

5. Di Mambro, T.; Guerriero, I.; Aurisicchio, L.; Magnani, M.; Marra, E. The yin and yang of current antifungal therapeutic strategies: how can we harness our natural defenses? Frontiers in Pharmacology 2019, 10, doi: 10.3389/fphar.2019.00080.

6. Hokken, M.W.J.; Zwaan, B.J.; Melchers, W.J.G.; Verweij, P.E. Facilitators of adaptation and antifungal resistance mechanisms in clinically relevant fungi. Fungal Genetics and Biology 2019, 132, 103254. https://doi.org/10.1016/j.fgb.2019.103254.

7. Kumar, P.; Singh, S.; Pratama, M.R.F. Synthesis of some novel 1H-indole derivatives with antibacterial and antifungal activity. Letters in Applied NanoBioScience 2020, 9, 961-967, https://doi.org/10.33263/LIANBS92.961967.

8. Hope, W.; Drusano, G.L.; Rex, J.H. Pharmacodynamics for antifungal drug development: an approach for acceleration, risk minimization and demonstration of causality. Journal of Antimicrobial Chemotherapy 2016, 71, 3008-3019, https://doi.org/10.1093/jac/dkw298. 
9. Lehrnbecher, T.; Bochennek, K.; Klingebiel, T.; Gastine, S.; Hempel, G.; Groll, A.H. Extended dosing regimens for fungal prophylaxis. Clinical Microbiology Reviews 2019, 32, https://doi.org/10.1128/CMR.00010-19.

10. Tada, R.; Latgé, J.P.; Aimanianda, V. Undressing the fungal cell wall/cell membrane--the antifungal drug targets. Curr Pharm Des 2013, 19, 3738-3747, https://doi.org/10.2174/1381612811319200012.

11. Liu, N.; Tu, J.; Dong, G.; Wang, Y.; Sheng, C. Emerging new targets for the treatment of resistant fungal infections. Journal of Medicinal Chemistry 2018, 61, 5484-5511, https://doi.org/10.1021/acs.jmedchem.7b01413.

12. Dranginis, A.M.; Rauceo, J.M.; Coronado, J.E.; Lipke, P.N. A biochemical guide to yeast adhesins: glycoproteins for social and antisocial occasions. Microbiology and Molecular Biology Reviews 2007, 71, 282-294, http://doi.org/10.1128/MMBR.00037-06.

13. Lesage, G.; Bussey, H. Cell wall assembly in Saccharomyces cerevisiae. Microbiology and Molecular Biology Reviews 2006, 70, 317-343, https://doi.org/10.1128/MMBR.00038-05.

14. Klis, F.M.; De Groot, P.; Hellingwerf, K. Molecular organization of the cell wall of Candida albicans. Medical Mycology 2001, 39, 1-8, https://doi.org/10.1080/mmy.39.1.1.8-0.

15. Karkowska-Kuleta, J.; Rapala-Kozik, M.; Kozik, A. Fungi pathogenic to humans: molecular bases of virulence of Candida albicans, Cryptococcus neoformans and Aspergillus fumigatus..Acta Biochimca Polonica 2009, 56, 211-224, https://doi.org/10.18388/abp.2009_2452.

16. de Groot, P.W.J.; Bader, O.; de Boer, A.D.; Weig, M.; Chauhan, N. Adhesins in human fungal pathogens: glue with plenty of stick. Eukaryotic Cell 2013, 12, https://doi.org/10.1128/EC.00364-12.

17. Nath, A. Prediction and molecular insights into fungal adhesins and adhesin like proteins. Computational Biology and Chemistry, 2019, 80, 333-340, https://doi.org/10.1016/j.compbiolchem.2019.05.001.

18. Beauvais, A.; Latgé, J.P. Special issue: fungal cell wall. Journal of Fungi 2018, 4, https://doi.org/10.3390/jof4030091.

19. Nobile, C.J.; Schneider, H.A.; Nett, J.E.; Sheppard, D.C.; Filler, S.G.; Andes, D.R.; Mitchell, A.P. Complementary adhesin function in C. albicansbiofilm formation. Current Biology 2008, 18, 1017-1024, https://doi.org/10.1016/j.cub.2008.06.034.

20. Sundstrom, P. Adhesins in Candida albicans. Current Opinion in Microbiology 1999, 2, 353-357, https://doi.org/10.1016/S1369-5274(99)80062-9.

21. Gurubasavaraj, M.P.; Charantimath, J.S. Recent advances in azole based scaffolds as anticandidal agents. Letters in Drug Design \& Discovery 2019, https://doi.org/10.2174/1570180815666180917125916.

22. Gale, C.; Finkel, D.; Tao, N.; Meinke, M.; McClellan, M.; Olson, J.; Kendrick, K.; Hostetter, M. Cloning and expression of a gene encoding an integrin-like protein in Candida albicans. Proceedings of the National Academy of Sciences 1996, 93, 357-361. https://doi.org/10.1073/pnas.93.1.357.

23. Hostetter, M.K. Integrin-like proteins in Candida spp. and other microorganisms. Fungal Genetics and Biology 1999, 28, 135-145, https://doi.org/10.1006/fgbi.1999.1165.

24. Hostetter, M.K. RGD-mediated adhesion in fungal pathogens of humans, plants and insects. Current Opinion in Microbiology 2000, 3, 344-348, https://doi.org/10.1016/S1369-5274(00)00101-6.

25. Jawhara, S.; Pluskota, E.; Verbovetskiy, D.; Skomorovska-Prokvolit, O.; Plow, E.F.; Soloviev, D.A.Integrin $\alpha \times \beta 2$ is a leukocyte receptor for Candida albicans and is essential for protection against fungal infections. The Journal of Immunology 2012, 189, 2468-2477, https://doi.org/10.4049/jimmunol.1200524.

26. Elhasi, T.; Blomberg, A. Integrins in disguise - mechanosensors in Saccharomyces cerevisiae as functional integrin analogues. Microbial Cell 2019, 6, 335-355, https://doi:10.15698/mic2019.08.686.

27. Sharma, R.K.; Singh, V.; Tiwari, N.; Butcher, R.J.; Katiyar, D. Synthesis, antimicrobial and chitinase inhibitory activities of 3-amidocoumarins. Bioorganic Chemistry 2020, 103700, https://doi.org/10.1016/j.bioorg.2020.103700.

28. Priyanka; Singh, V.; Ekta; Katiyar, D. Synthesis, antimicrobial, cytotoxic and E. coli DNA gyrase inhibitory activities of coumarinyl amino alcohols. Bioorganic Chemistry 2017, 71, 120-127, https://doi.org/10.1016/j.bioorg.2017.01.019.

29. Barot, K.P.; Jain, S.V.; Kremer, L.; Singh, S.; Ghate, M.D. Recent advances and therapeutic journey of coumarins: current status and perspectives. Medicinal Chemistry Research 2015, 24, 2771-2798, https://doi.org/10.1007/s00044-015-1350-8.

30. Singh, L.K.; Priyanka; Singh, V.; Katiyar, D. Design, synthesis and biological evaluation of some new coumarin derivatives as potential antimicrobial agents. Med Chem 2015, 11, 128-134, https://doi.org/10.2174/1573406410666140902110452.

31. Peng, X.M.; Damu, G.L.; Zhou, C. Current developments of coumarin compounds in medicinal chemistry. Curr Pharm Des 2013, 19, 3884-3930, https://doi.org/10.2174/1381612811319210013.

32. Prusty, J.S.; Kumar, A. Coumarins: antifungal effectiveness and future therapeutic scope. Molecular Diversity 2019, https://doi.org/10.1007/s11030-019-09992-x.

33. Al-Amiery, A.A.; Kadhum, A.A.H.; Mohamad, A.B. Antifungal activities of new coumarins. Molecules 2012, 17, 5713-5723, https://doi.org/10.3390/molecules 17055713. 
34. Cavasotto, C.N.; Phatak, S.S. Homology modeling in drug discovery: current trends and applications. Drug Discovery Today 2009, 14, 676-683, https://doi.org/10.1016/j.drudis.2009.04.006.

35. Arba, M.; Arfan, A.; Nuralifah.; Haerunnisah, O.W.; Kadidae, L. In silico exploration for aldose reductase (AR) inhibitors. Letters in Applied NanoBioScience 2020, 9, 956-960, https://doi.org/10.33263/LIANBS92.956960.

36. Laskowski, R.A.; MacArthur, M.W.; Moss, D.S.; Thornton, J.M. PROCHECK: a program to check the stereochemicalquality of protein structures. J Applied Crystallography 1993, 26, 283-291, https://doi.org/10.1107/S0021889892009944.

37. ACD/ChemSketch,Advanced Chemistry Development,Inc,Toronto,ON,Canada, 2010.

38. Morris, G.M.; Huey, R.; Lindstrom, W.; Sanner, M.F.; Belew, R.K.; Goodsell, D.S.; Olson, A.J. AutoDock4 and AutoDockTools4: Automated docking with selective receptor flexibility. Journal of Computational Chemistry 2009, 30, 2785-2791, https://doi.org/10.1002/jcc.21256.

39. Pettersen, E.F.; Goddard, T.D.; Huang, C.C.; Couch, G.S.; Greenblatt, D.M.; Meng, E.C.; Ferrin, T.E. UCSF Chimera-A visualization system for exploratory research and analysis. Journal of Computational Chemistry 2004, 25, 1605-1612, https://doi.org/10.1002/jcc.20084.

40. Müller, G.; Gurrath, M.; Kessler, H. Pharmacophore refinement of gpIIb/IIIa antagonists based on comparative studies of antiadhesive cyclic and acyclic RGD peptides. Journal of Computer-Aided Molecular Design 1994, 8, 709-730, https://doi.org/10.1007/BF00124017.

41. Dathe, M.; Nikolenko, H.; Klose, J.; Bienert, M. Cyclization increases the antimicrobial activity and selectivity of arginine- and tryptophan-containing hexapeptides. Biochemistry 2004, 43, 9140-9150, https://doi.org/10.1021/bi035948v.

42. Mittal, S.; Kaur, S.; Swami, A.; Maurya, I.K.; Jain, R.; Wangoo, N.; Sharma, R,K. Alkylated histidine based short cationic antifungal peptides: synthesis, biological evaluation and mechanistic investigations. RSC Advances 2016, 6, 41951-41961, DOI: 10.1039/c6ra05883c.

43. Mahindra, A.; Bagra, N.; Wangoo, N.; Khan, S.I.; Jacob, M.R.; Jain, R. Discovery of short peptides exhibiting high potency against Cryptococcus neoformans. ACS Medicinal Chemistry Letters 2014, 5, 315320, https://doi.org/10.1021/ml500011v. 